MATEC Web of Conferences 22,01042 (2015)

DOI: $10.1051 /$ matec conf/ 20152201042

(C) Owned by the authors, published by EDP Sciences, 2015

\title{
Method for Rapid Judgment of Faulty Stations of Multilateration System
}

\author{
Honggang $\mathrm{Wu}^{*}$, Yong Tang \& Xingbo Li \\ Research and Development Center, The Second Research Institute of CAAC, Chengdu, Sichuan, China
}

\begin{abstract}
An airport surface multilateration system based on the time difference of arrival (TDOA) positioning principle establishes equations to calculate the position of target through utilizing the TDOA of targets' answering signals to the ground receiving stations. The fault with any ground receiving station will result in a significant measuring error of signal's arrival time and have a lethal impact on the accuracy of positioning. A method for rapid judgment of station fault based on the smoothing filtering and judgment of deviation statistics is proposed. The target location will be firstly estimated through smoothing filtering of data, thus it is allowed to calculate the deviation of measuring position and the estimated position. When the deviation exceeds a certain threshold, it indicates that there is a large increase in the errors of single stations; then, the TDOA of each station can be calculated based on the estimated position, and the deviation can be calculated through comparison with the actually measured value of TDOA. For the stations with large deviation of TDOA as indicated by statistics, the station with the most deviations of large TDOA will be the station with problems. A simulation experiment for an airport indicates that the method can rapidly and effectively judge the faulty station.
\end{abstract}

Keywords: multilateration; smooth filtering; faulty station; simulation

\section{INTRODUCTION}

Over the past ten years, domestic and overseas air transportation has experienced a high-speed development and the role of air transportation is more and more important in the national economy. The increasingly busy air traffic and throughput capacity of airports increasing with each passing day will certainly give rise to a larger flight density, a small spacing and increasingly prominent aviation safety problems. Statistics show that $50 \%$ of air crashes happen in the landing stage. Runway incursion and collision among airplanes or between airplane and various vehicles on the airport surface often occur. The safety of airport surface and airport environment (for example, meteorological condition, visibility and so on) imposes more and more influence on the airport capacity and flight punctuality and has become the bottlenecks restricting the further enhancement of air transportation capacity. Thus the enhancement of monitoring and control for moving objects in airport has become an important part of the domestic and overseas air traffic control. For this purpose, foreign countries have invested a lot of manpower and money in carrying out relevant researches. The Advanced Surface Movement Guidance and Control Systems Manual published by ICAO in 2004 proposed a series of new concepts and requirements for the monitoring and control of airport surface [1]. The former monitoring requirement of airport surface was changed from the "be able to see, be seen" [2] mainly relying on the manual treatment by pilot and controller to the automatic guidance and control of runway, taxiway, parking apron and boarding gate of airplane on the airport. And the functions were increased which in- clude four major functions such as monitoring, route allocation, guidance and control, which could enhance the safety of airport in various environmental conditions, improve the airport capacity and solve the bottleneck problem of air transportation caused by the increasingly serious airport safety and delay. Therefore, it makes people realize that only relying on the surface monitoring radar system or a single monitoring system is hard to implement the comprehensive monitoring for the moving objects in the airport. Thus new researches on the surface monitoring and management system have been started overseas [3-5].

The multilateration technology [6-10] is a new airport surface monitoring technology proposed overseas. This technology gives full play to the $\mathrm{A} / \mathrm{C}$ model or $\mathrm{S}$ model response signal as well as the $\mathrm{S}$ model "squtter" signal of the standard configuration responder used for civil aircrafts, and applies the multi-point passive sensor receiving mode to determine the position of airplane or other moving objects. As this system is fully compatible with SSR radar and ADS-B downstream data transmission link, it can receive and decode the SSR code and S mode address of airplane. Thus, it has the capacities of the target identification and high precision positioning. The most important characteristic of this technology is giving full play to the existing standard airborne responder without the need to install other equipment of airborne aviation for completing the positioning monitoring. It is compatible with ADS-B technology and boasts high data updating rate (which is once per second, while the speed of secondary radar is at least once every four seconds), low system costs (which is $1 / 3$ less than that of existing SSR radar system), high positioning precision (which is within $50 \mathrm{~m}$ for the 


\section{MATEC Web of Conferences}

wide area air positioning and within $10 \mathrm{~m}$ for the ground positioning) and target identification capacity. As the passive receiving equipment has little interference for other systems, the antenna of receiving station is a simple omnidirectional antenna without a rotating mechanism. It is characterized by small volume, flexible station configuration and strong adaptability to the system monitoring coverage and so on.

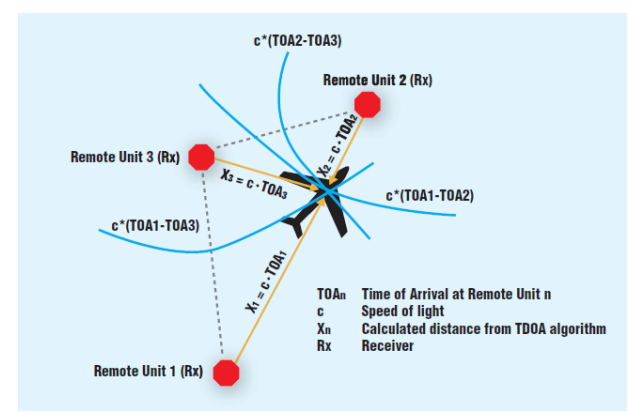

Figure 1. Working principle of multrlateration

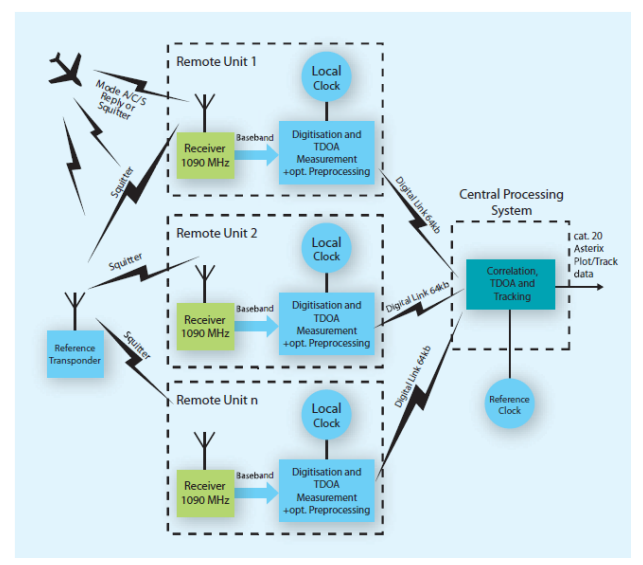

Figure 2. System chart of multilateration

The working principle of Multilateration system is shown in Figure 1. The system utilizes a number of passive radar receivers in distributing installation, which have the same working frequency for reception of secondary radar used in civil aviation to receive the response from the airborne standard $\mathrm{A} / \mathrm{C}$ mode or $\mathrm{S}$ mode responder in or the S mode "squtter" signal independently transmitted by the monitored airplane. The accurate position of the detected airplane is calculated with TDOA based on the hyperbolic curve (side) positioning principle. In addition, SSR code is extracted from an A mode signal or an airplane address code which is extracted from the $\mathrm{S}$ mode signal to provide airplane identification for subsequent treatments. Theoretically, the accurate two-dimensional position of airplane on the airport surface can be determined through three receiving stations and the coordinates of the three-dimensional position of airplane in the air can be determined with four receiving stations. In fact, due to airport terrain, shielding of buildings, coverage requirement of system monitoring, redundancy and other reasons, five to seven receiving stations are generally required. The functional block diagram of system is shown in Figure 2 .

In the technological development of multilateration, a number of passive location technical systems were proposed such as the RSSI system with multiple passive receiving stations to measure the intensity of target signal, the AOA system to measure the angle of target relative to the receiving station, the TOA system for direct measurement of time and so on. As the RSSI is prone to provide environmental disturbance, AOA receiving station must be configured with directional antenna. However, researches show that the TDOA utilizing time difference technology is a good multipoint passive location system. It utilizes the time difference of target signal to reach a number of passive sensors to determine the position of target measured. That is to say, use the hyperbolic curve cross positioning (two-dimensional) and the hyperbolic face cross positioning (three-dimensional) algorithm. The mathematical equation for calculation is shown in Figure 4. Target position can be made if we could accurately measure the time difference of each station related to the master station. As this system can be better used to eliminate the error with the target signal sources (responder) and the error with the wireless communication of signal, the existing experiments and application systems basically apply the TDOA system.

At present, the MLAT receiver has been installed in worldwide large and medium airports such as Heathrow, Frankfurt and Dallas and so on. [11] Although the multilateration has been applied in an extremely large scale, the majority of medium and small airports have installed fewer receiving stations of MLAT system. In this circumstance, in case of significant increase in detection time error of a station, it will have a lethal impact on the accuracy of final target positioning, and cause the danger of collision accident on the ground.

This paper proposes a method for judgement of faulty station based on the moving average filtering and judgment of deviation statistics and such method can rapidly and effectively judge whether there is a fault of significant increase in error of station at measurement, and determine the number of station. On this basis, the MLAT system can remove the data from the receiving station with fault, thus guaranteeing the system positioning accuracy can be controlled in the specified range.

\section{MATHEMATICAL MODEL OF MLAT POSI- TIONING}

The routes for targets to reach different receiving stations are different. That is to say, there is a differ- 
ence in distance. In respect of time, there is the time difference for the same answering signal to reach different receiving stations and it includes the spatial position information of target. Therefore, the target can be positioned through measuring the Time Difference of Arrival (TDOA) [12]. Assume that the vector of target's spatial position is $\mathbf{x}=\left[\begin{array}{lll}r_{x} & r_{y} & r_{z}\end{array}\right]^{T}$, there are $\mathrm{N}$ receiving stations. The position vector of the ith receiving station is $\mathbf{x}_{i}=\left[\begin{array}{lll}r_{x i} & r_{y i} & r_{z i}\end{array}\right]^{T}$, the arrival time of signal is $\mathrm{t}$, the distance from the target is $\mathrm{r}$, and $i=1,2, \ldots, N$, the measured value of arrival time is shown as follows:

$$
t_{m i}=t_{0}+\frac{r_{i}}{c}+n_{i}
$$

Wherein, $t_{0}$ is a time constant, $\mathrm{n}$ is the time measurement noise and $\mathrm{c}$ is the speed of light $\left(c=3 \times 10^{8} \mathrm{~m} / \mathrm{s}\right)$.

For any two receiving stations $\mathrm{p}$ and $\mathrm{q}$, the difference in arrival time of target's answering signal determines a hyperboloid and the receiving stations $p$ and $\mathrm{q}$ are at the focus of the hyperboloid. The difference in arrival time is shown as follows:

$$
\begin{aligned}
t_{p q} & =t_{p}-t_{q}=\frac{r_{2}-r_{1}}{c} \\
& =\frac{1}{c}\left[\sqrt{\left(r_{x}-r_{x p}\right)^{2}+\left(r_{y}-r_{y p}\right)^{2}+\left(r_{z}-r_{z p}\right)^{2}}-\sqrt{\left(r_{x}-r_{x q}\right)^{2}+\left(r_{y}-r_{y q}\right)^{2}+\left(r_{z}-r_{z q}\right)^{2}}\right]
\end{aligned}
$$

To combine the equations of $N$ receiving stations, the matrix form is shown as follows:

$$
\begin{aligned}
& \mathbf{T}_{m}=\mathbf{T}_{0}+\frac{1}{c} \mathbf{r}+\mathbf{n} \\
& \text { Wherein, } \\
& \mathbf{T}_{m}=\left[\begin{array}{llll}
t_{m 1} & t_{m 2} & \cdots & t_{m N}
\end{array}\right]^{T}, \\
& \mathbf{T}_{0}=\left[\begin{array}{llll}
t_{0} & t_{0} & \cdots & t_{0}
\end{array}\right]^{T}, \\
& \mathbf{r}=\left[\begin{array}{lll}
r_{1} & r_{2} \cdots & r_{N}
\end{array}\right]^{T}, \\
& \mathbf{n}=\left[\begin{array}{lll}
n_{1} & n_{2} \cdots & n_{N}
\end{array}\right]^{T} .
\end{aligned}
$$

The solution for equation set (3) is the target's positioning solution. In addition, the equation set is often a non-linear overdetermined equation set. Therefore, it is required to get the solution through iteration and approximation. At present, there are mainly two target positioning algorithms, that is, the close form solution represented by Chan Method [13] and the iterative algorithm represented by Taylor series expansion $[14,15]$.

\section{METHOD FOR JUDGMENT OF FAULTY RE- CEIVING STATION}

\subsection{Analysis of the influence of the error of a single} station on positioning accuracy

The size of target positioning error is mainly related with two factors: One is the measuring factor which reflects the influence of system measurement on accuracy and includes the measures of TDOA and the positioning measurement by the receiving station; another one is the geometry dilution of position (GDOP), which reflects the influence of the related geometric location relationship between the target and the receiving stations. When a time measurement error $\sigma_{t}$ is set, it is allowed to analyze the MLAT positioning error in the geometric layout of different stations [16].

In this situation, we apply the motion trail of airplane in an airport obtained with the high precision GPS (which is represented by "O" in Figure 3 ) as the real target position and assume that four remote receiving stations (which are represented by " $\oplus$ ") are set in the airport and the error of each station at measurement is $\sigma_{t}=5 n s$. The Chan Method can solve the target position (which is represented by “*”) of 4 stations obtained through the multilateration.

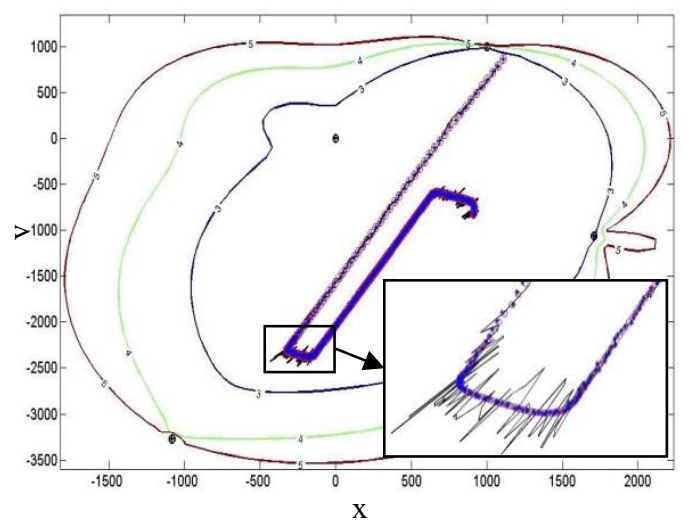

Figure 3. Multilateration positioning results of four stations and error influence of single station

In addition, it is also possible to draw the GDOP analysis results based on these conditions. The three outer curves in Figure 1 are equal precision curves with the precision $(1 \sigma)$ of $3 \mathrm{~m}, 4 \mathrm{~m}$ and $5 \mathrm{~m}$. The curve that centers on the changes of real target track in Figure 1 is the positioning results obtained when the error of station 4 at measurement is $20 \sigma_{t}$. Table 1 shows the comparison of positioning accuracy in case of the significant increase of different degrees in error of a single station at measurement at the different remote terminals. It can be seen from the table that the system's overall positioning precision will be increased significantly with the significant increase in error of a single station. Therefore, it is required to find the station with problems as soon as possible in case of a significant increase in error of a single station and eliminate the station's influence in resolving target position, so as to ensure the positioning accuracy.

Table 1. Precisions obtained at different stations and different errors at measurement (m), $\sigma_{t}=5 n s$ 


\section{MATEC Web of Conferences}

\begin{tabular}{|l|l|l|l|l|l|}
\hline $\begin{array}{l}\text { Error of } \\
\text { single station }\end{array}$ & $1 \sigma_{t}$ & $10 \sigma_{t}$ & $20 \sigma_{t}$ & $30 \sigma_{t}$ & $50 \sigma_{t}$ \\
\hline Station 1 & 1.82 & 10.51 & 23.46 & 35.03 & 57.76 \\
\hline Station 2 & 1.65 & 9.70 & 20.74 & 29.61 & 48.46 \\
\hline Station 3 & 1.74 & 9.26 & 18.34 & 28.79 & 46.82 \\
\hline Station 4 & 1.86 & 9.20 & 17.70 & 33.38 & 58.42 \\
\hline
\end{tabular}

\subsection{Method for judgment of faulty remote station}

Through the above analysis, we understand the extremely large influence of the significant increase in error of a single station on positioning precision. There are two critical problems: First one is the method to judge whether there is a significant increase in error of a single station; second one is the method to rapidly and accurately find out the station with problems in such case. Currently, there are few articles on the multilateration about such problems. Our thoughts are to firstly estimate the real position of target through the smoothing filtering of data to calculate the deviation of measured position from the estimate position. The deviation exceeding a threshold indicates the significant increase in error of a single station; then, its TDOA to other stations can be calculated based on the estimated position, the deviation will be calculated through comparison with the actually measured value of TDOA and the stations with a large deviation of TDOA will be found out through statistics. The station causing the most deviation of large TDOA will be a station with problems. The specific method is shown as follows.

\subsubsection{Smoothing filtering}

There are many methods for conducting smoothing filtering of data, such as Least Squares, Least Mean Square and Kalman filtering and so on. The smoothing filtering is actually a low-pass filter which is used to suppress noise and make the target track smoother. As the focus of this paper is on the optimal selection of the multilateration station, only a simple and practical smoothing method is applied here, that is, the moving average filtering. Assume that the width of filtering window is $\mathrm{W}$, and ones $(W, 1)$ represents the array of width in which $\mathrm{W}$ is 1 , the coordinates of track after the smoothing filtering are shown as follows:

$\overline{\mathbf{r}}_{x}=\hat{\mathbf{r}}_{\mathbf{x}} * \frac{1}{W} \operatorname{ones}(W, 1)$,

$\overline{\mathbf{r}}_{y}=\hat{\mathbf{r}}_{y} * \frac{1}{W} \operatorname{ones}(W, 1)$

Wherein, $\overline{\mathbf{r}}_{x}, \overline{\mathbf{r}}_{y}$ are the coordinates of track after smoothing filtering, and $\hat{\mathbf{r}}_{x}, \hat{\mathbf{r}}_{y}$ are the coordinates of track calculated with the multilateration. As the relative altitude of airplanes on the ground is 0 , it is not required to conduct filtering for $\mathbf{r}_{z}$.

\subsubsection{Judgment of significant increase in error of station}

For simplification, only two consumptions are con-

sidered: $H 0$ is that there is no station with a significant increase in error at measurement; $H 1$ is that there is a station with a significant increase in error at measurement; The theoretical value of positioning precision $\sigma_{x y}$ can be obtained within an effective coverage range through analysis of error at measurement $\sigma_{t}$ and GDOP. For example, all the airplane track points in Figure 1 are within the range covered by curves with the precision of $3 \mathrm{~m}$, etc. Therefore, it can be considered that $\sigma_{x y}=3$. However, we know that it is very unlikely for the positioning deviation of airplane at a moment to exceed $3 \sigma_{x y}$. Therefore, if a positioning deviation exceeds $3 \sigma_{x y}$, we can conclude that there is a significant increase in error of a single station. Although this judgment is not $100 \%$ accurate, the measured value of positioning at all moments and the distance deviation of smoothing value can be obtained with the following equation:

$$
\hat{e}(t)=\sqrt{\left[\bar{r}_{x}(t)-\hat{r}_{x}(t)\right]^{2}+\left[\bar{r}_{y}(t)-\hat{r}_{y}(t)\right]^{2}}
$$

Open a time window with the width of $\mathrm{M}(\mathrm{M}$ can be $1,2,3 \ldots)$ at t. If $k=M, M-1, \ldots, 0$ in $\hat{e}(t-k)$, and there are $M_{e t}$ exceeding $3 \sigma_{x y}$, the decision statistics $R E_{t}=M_{e t} / M$ can be used to judge whether there are stations with a significant increase in error at measurement, and it is shown as follows:

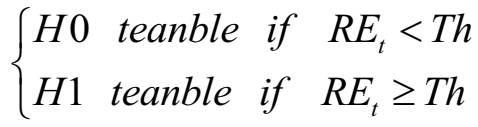

Wherein, Th is the judgment threshold. For example, $T h$ can be 0.1 or 0.2 . The larger $T h$ is, the stricter the judgment conditions will be.

\subsubsection{Determination of station with problems}

At $\mathrm{t}$, when the judgment is $H 1$, select the smoothing values of the positions $\bar{r}_{x}, \bar{r}_{y}$ corresponding to errors in the time window $\mathrm{M}$ with the $M_{e t}$ exceeding $3 \sigma_{x y}$ and calculate the time difference $\bar{t}_{p q}$ for it to reach each station with equation (2), and compare it with the actually measured TDOA $\hat{t}_{p q}$, as shown in the equation as follows:

$$
D t_{p q}=\left|\bar{t}_{p q}-\hat{t}_{p q}\right|, \quad p, q=1, \ldots, N
$$

Wherein $\mathrm{N}$ is the number of station. Find out the $N / 2$ largest value in each group of $D t_{p q}$, judge the number of station generating such $D t_{p q}$, and perform accumulation to obtain the number of station with problems.

\section{SIMULATION EXPERIMENT}

\subsection{Smoothing filtering}

In order to verify whether the value after the smoothing filtering can be used as the estimation of true value, we still apply a high precision GPS to obtain 
ICETA 2015

Table 3. Accuracy of judgment of significant increase in error of single station and the accuracy of judgment of station with problems

\begin{tabular}{|l|l|l|l|l|l|l|l|l|l|l|}
\hline & \multicolumn{6}{|l|}{$\begin{array}{l}\text { Accuracy of judgment of significant } \\
\text { increase in error of single station (\%) }\end{array}$} & \multicolumn{2}{l}{$\begin{array}{l}\text { Accuracy of judgment of station with } \\
\text { problems (\%) }\end{array}$} \\
\hline Error of Single Station & $10 \sigma_{t}$ & $20 \sigma_{t}$ & $30 \sigma_{t}$ & $50 \sigma_{t}$ & $10 \sigma_{t}$ & $20 \sigma_{t}$ & $30 \sigma_{t}$ & $50 \sigma_{t}$ \\
\hline Station 1 & 97.14 & 100 & 100 & 100 & 79.43 & 87.14 & 91.71 & 94.14 \\
\hline Station 2 & 99.20 & 99.71 & 100 & 100 & 85.28 & 91.19 & 94.43 & 96.29 \\
\hline Station 3 & 93.74 & 97.32 & 99.64 & 100 & 86.10 & 94.61 & 96.36 & 97.79 \\
\hline Station 4 & 92.86 & 94.40 & 97.29 & 99.07 & 81.29 & 88.23 & 92.29 & 94.86 \\
\hline
\end{tabular}

the airplane track as the true value and set the basic time measurement errors of four remote stations of airport as $\sigma_{t}=5 n s$.We select different stations to change their errors at measurement and have conducted 100 Monte Carlo simulation experiments to calculate the positioning precision after smoothing filtering.

The width of smoothing filtering window is $W=9$. Figure 4 shows the comparison of results after the smoothing filtering in case of $30 \sigma_{t}$ error at station 4 . Obviously, the fluctuation of data after filtering (which is represented by "*”) has significantly decreased, and the data is closer to the real position. Table 2 also reflects such result. In particular, the comparison in Table 1 shows that the positioning precision after application of the smoothing filtering due to the significant increase in error of single station is increased by several times.

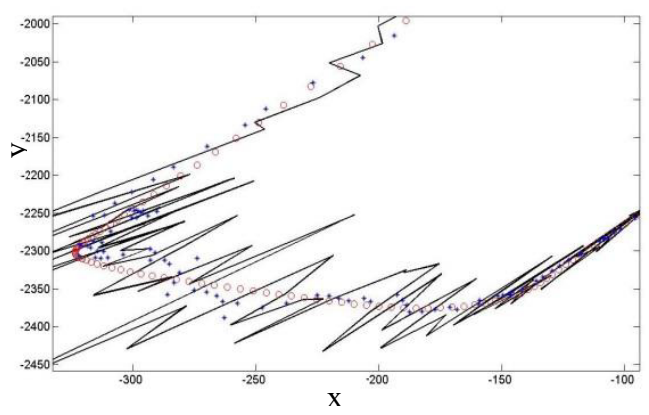

Figure 4. Effects after smoothing filtering of track

Table 2. Precision obtained through smoothing filtering at different stations and different errors at measurement (m), $\sigma_{t}=5 n s$

\begin{tabular}{|l|l|l|l|l|l|}
\hline $\begin{array}{l}\text { Error of single } \\
\text { station }\end{array}$ & $1 \sigma_{t}$ & $10 \sigma_{t}$ & $20 \sigma_{t}$ & $30 \sigma_{t}$ & $50 \sigma_{t}$ \\
\hline Station 1 & 1.79 & 4.14 & 7.89 & 13.74 & 23.03 \\
\hline Station 2 & 1.95 & 3.98 & 7.45 & 11.63 & 21.09 \\
\hline Station 3 & 1.87 & 2.65 & 6.74 & 10.67 & 19.65 \\
\hline Station 4 & 1.93 & 4.32 & 7.61 & 14.38 & 25.94 \\
\hline
\end{tabular}

4.2 Judgment of significant increase in error of single station and determination of station with problems

According to the method as described earlier, we set $N=4, \sigma_{x y}=3, M=5$ and $T h=0.2$ as well as other simulation conditions to be the same as those in section 4.1. We have conducted 100 Monte Car- losimulation experiments, and made statistics of the accuracy of judgment of significant increase in error of single station and the accuracy of judgment of station with problems.

The conclusion that we can obtain from Table 3 is that the application of the method as described in the paper can obtain a high accuracy of judgment of significant increase in error of single station and a high accuracy of judgment of station with problems. In an actual application, the station judgment accuracy of eighty percent of stations is sufficient to find out the remote station with a large error at measurement in a very short time, thus it can achieve the optimal selection of multilateration station. In addition, it is worth mentioning that, in case of significant increase in errors of different stations, the judgment accuracies we can obtain are different, which indicates that the geometric position of station has certain influence on the performance of the method as described in this paper.

\section{CONCLUSION}

This paper proposes a method for the optimal selection of multilateration remote stations on the airport surface based on smoothing filtering and the strategy of statistics judgment of deviation, and this method can rapidly determine the faulty station in a very short time. A lot of Monte Carlo simulation experiments have verified the effectiveness of the algorithm in this paper. However, it is still required to further discuss the theoretical derivation problems in relation to the performance of the method as described in this paper. And the solutions in the case of significant increase in errors of multiple stations and the application of track filtering algorithm with better performance and so on will be shown in the further study.

\section{ACKNOWLEDGEMENT}

This paper is supported by the National Natural Science Foundation of China (GN: U1333202, U1233103). 


\section{MATEC Web of Conferences}

\section{REFERENCES}

[1] International Civil Aviation Organization (ICAO). 2004. Doc.9830-AN/452, Advanced surface movement guidance and control systems (A-SMGCS) Manual.

[2] International federation of air traffic controllers' associations, A-SMGCS Implementation in Europe, 2003.

[3] M. Röder. 2005. State of the Art in A-SMGCS, EMMA Project Partners.

[4] Demostration Facilities For Aerodrome Movement Management (DEFAMM), European Commission DG VII: Air Transports/Task 4.3.1/44.

[5] FAA. 1988. Airport Surface Operations Safety Plan to Prevent Ruanway Incursions an Improve Surface Operations. Ruanway Incursion Program Office (ATO-1-2).

[6] Hiromi Miyazaki, Noboru Miyoshi. Electronic Navigation Research Institute, Tokyo, Japan evaluation of ADS-B using modes extended squitter at Enri.

[7] Litchford, George B. Passive ATCRBS using signals of remote SSR.US patent 4115771/1978.

[8] Alsup, James M., Jelks, Edward C. Fix-tracking system. US patent 5191342/1993.

[9] Simuth, Alexander E. Lee, Derrick D. Multilateration auto-calibration and position error correction. US patent 6094169/2000.

[10]Winner, Karl; Khen; Benjamin. Vehicle Surveillance System. US patent $6816105 / 2004$.

[11] Lv Xiaoping. 2006. Discussion on the application of MDS technology in the civil aviation in China. Air Traffic Management, (9): 4-11.

[12]Harry B. Lee. 1975. Accuracy limitations of hyperbolic multilateration systems. IEEE Trans. on AES, AES-11(1): 16-29.

[13]Chan Y T, Ho K C. 1994. A simple and efficient estimator for hyperbolic location. IEEE Trans. On Signal Processing, SP-42(8): 1905-1915.

[14]Deng Ping and Fan Pingzhi. 2004. Cooperative localization based on chan method and taylor series expansion. Electronics and Information Journal, 26(1): 41-46.

[15]Foy W. H. 1976. Position location solutions by Taylor-series estimation. IEEE Trans. On AES, AES-12(2): 187-163.

[16]Deng Ping \& Yu Jianli. 2005. GDOP performance analysis of cellular location system. Journal of Southwest Jiaotong University, 42 (2): 184-188. 